\title{
Amotivation is associated with reduced ventral striatum volumes independently of dopamine receptor blockade by anti-psychotics in elderly schizophrenia patients
}

\author{
Fernando Caravaggio $^{1,2}$, Philip Gerretsen ${ }^{1,2}$, Gary Remington $^{1,2}$, Ariel Graff-Guerrero ${ }^{1,2}$ \\ ${ }^{I}$ Department of Psychiatry, University of Toronto, Canada, ${ }^{2}$ Centre for Addiction and Mental Health, Canada
}

Motivational deficits are prevalent in patients with schizophrenia persist despite antipsychotic treatment and predict longterm outcomes. Evidence suggests that patients with greater amotivation have smaller ventral striatum (VS) volumes. We wished to replicate this finding in a sample of older chronically medicated patients with schizophrenia. Using structural imaging and positron emission tomography (PET) we examined whether amotivation uniquely predicted VS volumes beyond the effects of striatal dopamine (DA) D2/3 receptor (D2/3R) blockade by antipsychotics. Data from 41 elderly schizophrenia patients (mean age 60.2711 female) were reanalyzed from previously published imaging data. We constructed multivariate linear step-wise regression models with VS volumes as the dependent variable and various sociodemographic and clinical variables as the initial predictors age gender total brain volume and antipsychotic striatal D2/3R occupancy. Amotivation was included as a subsequent step to determine any unique relationships with VS volumes beyond the contribution of the covariates. In a reduced sample $(n=36)$ general cognition was also included as a covariate. Amotivation uniquely explained $8 \%$ and $6 \%$ of the variance in right and left VS volumes respectively (right $\mathrm{B}=-.38 \mathrm{t}=$ $-2.48 \mathrm{p}=.01$ left $\mathrm{B}=-.31 \mathrm{t}=-2.17 \mathrm{p}=.03$ ). Considering cognition amotivation levels uniquely explained $9 \%$ of the variance in right VS volumes $(\mathrm{B}=-.43 \mathrm{t}=-.26 \mathrm{p}=.03)$. We replicate and extend the finding of reduced VS volumes with greater amotivation. We demonstrate this relationship uniquely beyond the potential contributions of striatal D2/3R blockade by antipsychotics. Elucidating the structural correlates of amotivation in schizophrenia may help develop treatments for this presently irremediable deficit. 